\title{
Desigualdades na mortalidade por doenças cardiovasculares em pequenos municípios
}

\author{
Inequalities in mortality from cardiovascular diseases \\ in small municipalities
}

Cássia Regina Gotler Medeiros ${ }^{1}$

Stela Nazareth Meneghel ${ }^{1}$

Tatiana Engel Gerhardt ${ }^{1}$

${ }^{1}$ Programa de Pósgraduação em Enfermagem, Universidade Federal do Rio Grande do Sul (UFRGS). Rua Frederico Bertoldo Schneider 1039, B

Universitário. 95900-000

Lajeado RS

cassiargm@terra.com.br
Abstract The scope of this ecological study was to identify mortality from cardiovascular disease in the municipalities of the 16th Health Region of Rio Grande do Sul between 2003 and 2007. It looked at differences among them, sex and age distribution and related the mean coefficient of mortality during the period to socioeconomic variables of investment, healthcare coverage and access, seeking to identify a few factors that might be significant to explain these differences. Correlation and multiple regression were analyzed. The results indicated that in counties with up to 5000 inhabitants the Family Health Strategy coverage may be a better protective factor than in larger municipalities, and per capita income has less influence on mortality. A direct relationship was found between hospital admissions for cardiovascular disease and mortality, especially in the larger municipalities. Multiple regression showed health costs per inhabitant/year as the factor most closely related to increased mortality. It was concluded that the variables selected explain partially the differences in mortality caused by cardiovascular disease in a similar group of municipalities of a regional health area in southern Brazil. Key words Cardiovascular disease, Inequalities in health, Health assessment, Public health policies, Socioeconomic factors
Resumo Estudo ecológico que teve por objetivo identificar a mortalidade por doenças cardiovasculares nos municípios da $16^{a}$ Regional de Saúde (RS), no período de 2003 a 2007, verificando diferenças entre eles, a distribuição por sexo e idade e relacionando o coeficiente médio de mortalidade do período com variáveis socioeconômicas, de investimento, de cobertura e de acesso em saúde, buscando identificar alguns fatores que poderiam ser significativos para explicar estas diferenças. Foi realizada análise de correlação e regressão múltipla. Os resultados indicaram que em municípios com até 5000 habitantes a cobertura da Estratégia Saúde da Família pode representar um fator protetor melhor do que nos municipios maiores e a renda per capita tem menor influência na mortalidade. Encontrou-se relação direta entre as internações por doenças cardiovasculares e a mortalidade, principalmente nos municipios maiores. A regressão múltipla mostrou a despesa em saúde por habitantelano como o fator mais relacionado com o aumento da mortalidade. Concluiu-se que as variáveis selecionadas no estudo explicam apenas parcialmente as diferenças na mortalidade por doenças cardiovasculares em um grupo de municípios similares de uma regional de saúde no sul do Brasil.

Palavras-chave Doenças cardiovasculares, Desigualdades em saúde, Avaliação em saúde, Políticas públicas de saúde, Fatores socioeconômicos 


\section{Introdução}

O envelhecimento da população, consequência da queda nas taxas de fertilidade e do aumento da expectativa de vida, incide fortemente no perfil epidemiológico da população e na demanda pelos serviços de saúde. Este envelhecimento leva a um aumento das patologias crônicas e das comorbidades, sendo que muitos sistemas de saúde enfrentam problemas associados à pobreza $\mathrm{e}$ exclusão social ${ }^{1}$. Mendes ${ }^{2}$ faz uma análise da situação de saúde no Brasil referindo a transição demográfica acelerada, em processo de rápido envelhecimento, que traz crescente incremento das condições crônicas.

O Brasil tem vivenciado mudanças no perfil epidemiológico, sendo que a partir da década de 60, as doenças crônicas não transmissíveis (DCNT) passaram a ocupar o primeiro lugar como causas de mortalidade geral. Dentre estas, as cardiovasculares, principalmente as isquêmicas do coração, as hipertensivas e as cerebrovasculares. As quais também são responsáveis por grande parte das internações e incapacidades, demandando alto custo para o SUS. Observa-se nos últimos anos uma pequena redução na mortalidade por doenças cardiovasculares (DCV), especialmente as cerebrovasculares, mas a taxa continua elevada, demonstrando a pouca efetividade das políticas públicas na atenção a este problema ${ }^{3}$.

A 16a Regional de Saúde localiza-se na bacia do Rio Taquari, na encosta inferior do nordeste do Rio Grande do Sul (RS), é composta por 42 municípios e sua população é de 363.964 habitantes ${ }^{4}$, tendo como sede o município de Lajeado. É expressivo o número de municípios de pequeno porte nesta região, sendo que 36 deles tem menos de 15 mil habitantes, o que se constitui em um grande desafio para a implementação das diretrizes do SUS, pois estes têm grande dependência externa para atender a população e garantir o princípio da integralidade. Conforme se observa, as dificuldades vão desde a estrutura física deficiente, baixa capacidade de reter e qualificar trabalhadores em saúde, pouca qualificação da gestão, até a forma de organização e funcionamento das redes de atenção à saúde.

No Rio Grande do Sul as DCV representaram 30,2\% dos óbitos em 2007. Na 16a Regional de Saúde, as DCV também ocupavam em 2007 o primeiro lugar como causa de mortalidade geral $(33,1 \%)$ e segundo lugar como causa de internações pelo SUS $(14,74 \%)$. O coeficiente de mortalidade (CM) pelas principais DCV no Brasil em
2007 foi de 109,93, enquanto que no RS foi de 139,17 e na $16^{a}$ Regional foi de $141,42^{5}$.

Em relação à estrutura da rede assistencial regional, a cobertura da Estratégia Saúde da Família (ESF) representava em 2010 aproximadamente $43 \%$. Esta cobertura era realizada por meio de 58 ESF, implantadas em 37 municípios, sendo que 27 com $100 \%$ de cobertura. Somente 5 municípios ainda não haviam implantado a ESF em $2010^{5}$. Esta estratégia possibilita ampliar as ações no território, permitindo um maior conhecimento das demandas destas populações. A regional tem como referência na média e alta complexidade em DCV, o Serviço de Cardiologia do Hospital Macrorregional localizado em Lajeado.

Entender esta complexa rede de atenção à saúde, suas deficiências e potencialidades, é um desafio que se impõe, na medida em que os serviços buscam qualificar esta atenção, a fim de impactar positivamente nos resultados de saúde, especialmente, no foco deste estudo, nos indicadores de mortalidade por DCV.

Diversos estudos têm sido realizados a respeito das doenças crônicas, principalmente enfatizando fatores de risco e indicadores de morbimortalidade ${ }^{6-8}$. Percebe-se a necessidade de ampliar o enfoque dado a esta problemática, considerando as especificidades regionais e de diferentes grupos sociais, incluindo aqui as questões pertinentes aos determinantes sociais em saúde, acesso e integralidade.

A articulação entre o estudo de causas de mortes evitáveis com a análise das desigualdades sociais e do acesso é uma possibilidade de abordagem do monitoramento da efetividade frente ao importante papel atribuído às condições de vida. O indicador denominado "Brechas Redutíveis de Mortalidade", que mede o risco de morte atribuível às condições de vida, desenvolvido pela Organização Pan-Americana de Saúde (OPS), pode revelar indiretamente a efetividade dos serviços de saúde quando se compara os indicadores de mortalidade por causas evitáveis entre regiões com características sociais semelhantes?

Embora o volume de riqueza seja fundamental para melhores condições de vida e de saúde de uma sociedade, nem sempre há uma correlação constante entre os macro-indicadores de riqueza e os indicadores de saúde, pois existem países com um Produto Interno Bruto (PIB) total ou per capita superior a outros e, no entanto, possuem indicadores de saúde menos satisfatórios. Estudar esta cadeia de mediações pode identificar os pontos mais sensíveis para que intervenções sobre iniquidades em saúde possam provocar maior impacto ${ }^{10}$. 
O principal desafio dos estudos sobre as relações entre determinantes sociais e saúde consiste em estabelecer uma hierarquia de determinações entre os fatores mais gerais de natureza social, econômica, política e as mediações através das quais esses fatores incidem sobre a situação de saúde de grupos e pessoas, já que a relação de determinação não é uma simples relação direta de causa-efeito ${ }^{10-11}$.

As condições socioeconômicas, culturais e ambientais de uma dada sociedade geram uma estratificação econômico-social dos indivíduos e grupos da população, conferindo-lhes posições sociais distintas, as quais por sua vez provocam diferenciais de saúde. Pode-se afirmar que a distribuição da saúde e da doença em uma sociedade não é aleatória, estando associada à posição social, que por sua vez define as condições de vida e trabalho dos indivíduos e grupos ${ }^{11}$.

Alguns estudos comprovam esta associação. Os dados da Pesquisa Nacional de Amostra por Domicílio (PNAD) de 2003 foram estudados no que se refere à prevalência de doenças crônicas. A escolaridade, ajustada para sexo, idade, cor da pele e macrorregião, mostrou que as pessoas com 0-3 anos de estudo tiveram quase duas vezes mais doenças que aquelas com mais de oito anos de estudo $^{12}$.

Da Costa et al. ${ }^{13}$ avaliaram fatores socioeconômicos, comportamentais e nutricionais para hipertensão na cidade de Pelotas, em um estudo transversal realizado em 2000. Comparadas com uma renda maior que 10 salários mínimos, todas as outras faixas salariais mostraram prevalência duas vezes maior de hipertensão, não tendo sido encontrada associação com escolaridade.

Outra pesquisa investigou a equidade vertical no Brasil, com base no rendimento auferido pelas pessoas, extraindo os dados da PNAD 98, dividindo as variáveis de saúde em três grupos: Necessidades de cuidados com a saúde (autoavaliação do estado de saúde, se esteve acamado nas duas últimas semanas, se tem doença crônica); Seguro saúde (plano de saúde, valor da mensalidade, cobertura do plano e plano odontológico) e Uso ou consumo dos serviços de saúde (procurou serviços de saúde nas duas últimas semanas e qual o motivo principal da procura $)^{13}$.

Esse estudo revelou que existem desigualdades em relação à presença ou ausência de doenças crônicas em populações pobres. As morbidades mais fáceis de serem percebidas como dor de coluna, artrite ou reumatismo, são relatadas frequentemente pelos de menor poder aquisitivo, enquanto que as doenças que precisam de exames para detectá-las, como câncer, diabete e hipertensão, são mais relatadas pelos que estão no topo da distribuição de renda, revelando maior oportunidade destes últimos no acesso aos serviços de saúde e não na maior probabilidade de adquirir tais afecções. Observou-se também desigualdade no consumo dos serviços de saúde pelos menos privilegiados, ressaltando-se que o motivo da procura destes é devido a doenças, enquanto que os mais privilegiados procuram os serviços para prevenção e exames de rotina. Verificou-se que as pessoas mais pobres têm pior acesso, necessitam de maiores cuidados com a saúde, mas consomem menos os serviços, agravando o quadro de desigualdade de renda, gerando uma saúde mais precária.

Outro achado deste estudo observou que as chances de procurar serviços de saúde crescem de acordo com o aumento da densidade populacional, o que pode ser explicado pela escassez desses serviços nas áreas rurais e de menor densidade populacional. Também se verificou que populações com maior escolaridade e acesso aos serviços públicos (água, luz, esgoto, coleta de lixo) tem aumentadas as chances de procurar os serviços de saúde.

Os estudos epidemiológicos sobre mortalidade por DCV têm se concentrado na comparação entre regiões, grandes cidades ou regionais dentro dos estados ${ }^{8,12,14}$. Questiona-se como compreender as diferenças existentes entre pequenos municípios de uma mesma região. Existem diferenças significativas nos indicadores de mortalidade por DCV? Quais variáveis estão relacionadas aos resultados destes indicadores?

O objetivo deste estudo foi identificar o CM pelas principais DCV nos municípios da $16^{\mathrm{a}} \mathrm{Re}-$ gional de Saúde, no período de 2003 a 2007, verificando diferenças entre eles, a distribuição por sexo e idade na região e relacionando o coeficiente médio de mortalidade (CMM) do período com algumas variáveis socioeconômicas, de investimento, de cobertura e de acesso em saúde, buscando identificar fatores significativos para explicar diferenças entre os municípios.

As variáveis relacionadas foram: Índice de Desenvolvimento Socioeconômico para o Rio Grande do Sul (IDESE), Proporção de população rural, Renda per capita, Taxa de analfabetismo, Proporção de população acima de 25 anos com mais de 12 anos de escolaridade, Distância em $\mathrm{Km}$ entre o município e o serviço de referência em cardiologia, Cobertura da ESF, Proporção de internações por doenças do aparelho circulatório (DAC), Percentual de investimento 
municipal em saúde e Despesa anual por habitante em saúde.

Este estudo é uma aproximação inicial a um contexto onde se pretende desenvolver uma abordagem qualitativa que analise em profundidade as dinâmicas locais em pequenos municípios, que possam estar resultando em diferenças nos indicadores de morbimortalidade por DCV, tendo como unidade de análise as redes de cuidado aos portadores de DCV.

\section{Delineamento do estudo}

Trata-se de um estudo observacional de base populacional e transversal, do tipo agregado, denominado ecológico.

A população em estudo foram os 42 municípios que compõe a 16a Regional de Saúde - Lajeado (RS). O indicador selecionado como desfecho foi o coeficiente de mortalidade por doenças do aparelho circulatório (capítulo IX do CID-10), discriminado segundo três grupos de causas (CID-BR-10): doenças isquêmicas do coração, doenças hipertensivas e doenças cerebrovasculares, relativas ao período de 2003 a 2007, obtidas no Sistema de Informações de Mortalidade $(\mathrm{SIM})^{5}$. Para o denominador utilizou-se a projeção intercensitária para cada ano em estudo, tendo como fonte os dados demográficos do Instituto Brasileiro de Geografia e Estatística (IBGE) ${ }^{4} \mathrm{e}$ como multiplicador 100 mil habitantes. Verificouse o comportamento do Coeficiente de Mortalidade por DCV na região, segundo sexo e idade.

O coeficiente médio de mortalidade (CMM) foi calculado para cada município, a fim de correlacionar com as variáveis independentes selecionadas:

- IDESE: índice sintético que abrange um conjunto de doze indicadores sociais e econômicos classificados em quatro blocos temáticos: educação (taxa de abandono no ensino fundamental, taxa de reprovação no ensino fundamental, taxa de atendimento no ensino médio, taxa de analfabetismo de pessoas de 15 anos e mais de idade); renda (geração de renda-PIB per capita, apropriação de renda); condições de saneamento e domicílio (percentual de domicílios atendidos com esgoto sanitário, média de moradores por domicílio); e saúde (percentual de crianças com baixo peso ao nascer, taxa de mortalidade de menores de cinco anos e esperança de vida ao nascer). Este índice varia de zero a um: baixo (até 0,499), médio (entre 0,500 e 0,799), ou alto (maio- res ou iguais a 0,800), segundo a Fundação de Economia e Estatística. Os dados eram relativos a $2006^{15}$.

- Distância em Km entre o município e o serviço de referência em cardiologia, segundo a Associação Brasileira de Concessionárias de Rodovia ${ }^{16}$.

- Proporção de cobertura da ESF por município: obtida nos indicadores da atenção básica do Datasus/Ministério da Saúde (MS), relativa a $2007^{5}$.

- Proporção de população rural e renda per capita: esta representa a razão entre o somatório da de todos os indivíduos e o número total destes. A renda per capita de cada indivíduo é definida como a razão entre a soma da renda de todos os membros da família e o número de membros da mesma. Valores expressos em reais de $1^{\circ}$ de agosto de 2000. Os dois indicadores foram obtidos no site da Confederação Nacional dos Municípios ${ }^{17}$.

- Taxa de analfabetismo: proporção de analfabetismo na população de 7 anos e mais, obtida no Datasus/MS, referente a $2000^{5}$.

- Proporção de população acima de 25 anos com mais de 12 anos de escolaridade: obtida no site do Programa das Nações Unidas para o Desenvolvimento (PNUD), dados do Censo Demográfico de $2000^{18}$.

- Proporção de internações por DAC: obtida no capítulo IX do CID-10, referente ao período 2003-2007, no Datasus/MS ${ }^{5}$.

- Percentual de investimento municipal em saúde e despesa anual por habitante em saúde: obtidos nos Cadernos de informações de saúde RS-versão maio/2010, orçamentos públicos, no Datasus/MS, referentes a $2007^{5}$.

Utilizou-se para o cálculo dos indicadores e confecção das tabelas o aplicativo Tabwin, do MS e o software Excel. Para as análises de correlação e regressão múltipla, utilizou-se o software SPSS, versão 15, para o conjunto dos 42 municípios e também estratificando em municípios com até 5000 hab., de 5001 a 15 mil e com mais de 15 mil hab. Esta estratificação foi intencional, pois um baixo número de agravos em municípios com populações muito pequenas pode representar altos coeficientes, provocando equívocos na comparação entre eles.

Pode-se considerar como limitação deste estudo a utilização de bancos de dados secundários, uma vez que a qualidade das informações não apresenta $100 \%$ de confiabilidade.

Por ser um estudo que utilizou dados de domínio público, não foi necessário submetê-lo a aprovação de Comitê de Ética em Pesquisa. 


\section{Resultados}

Observa-se na região uma tendência de queda na mortalidade por DCV, no período estudado (Gráfico 1). Esta é uma tendência já indicada por dados do IDB [descrever esta sigla] ${ }^{3}$ a nível nacional. O coeficiente de mortalidade foi de 170,81 em 2003 e 141,42 em 2007, representando uma queda de $17 \%$.

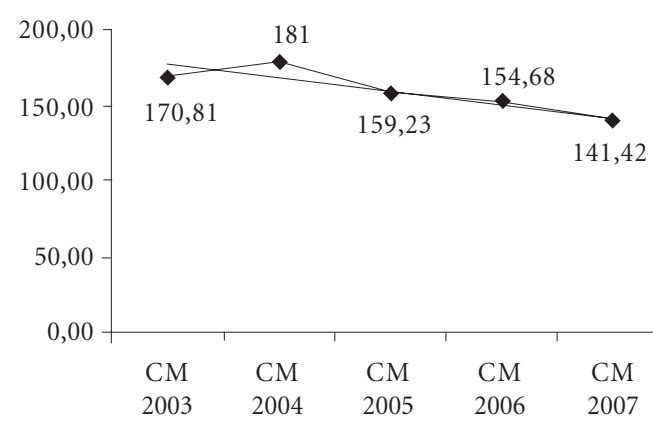

Gráfico 1. Coeficiente de Mortalidade por doenças cardiovasculares na $16^{\text {a }}$ Regional de Saúde/RS, no período de 2003 a 2007.

Fonte: SIM/Datasus/MS. CM: coeficiente de mortalidade por DCV
Comparando o CMM por DCV nos municípios da região, após estratificação por população, verificam-se diferenças importantes entre eles (Tabela 1). Nos municípios com até 5000 habitantes, a diferença no CMM foi de 4,8 vezes entre o menor e o maior coeficiente. Entre 5001 a 15 mil foi de 2,1 vezes e nos municípios com mais de 15 mil habitantes foi de 1,5 vezes. Percebe-se que quanto menor o município, mais acentuadas são as diferenças nos coeficientes, no entanto, observando o número absoluto de óbitos, a maior diferença está entre os municípios de 5001 a 15 mil habitantes, ou seja, morreram 3 vezes mais pessoas no município com maior coeficiente dentro desta estratificação.

Investigando a mortalidade por DCV em relação ao sexo, encontrou-se preponderância de óbitos femininos, que representaram $51,7 \%$ do total no período. No entanto, é relevante observar que $48 \%$ dos óbitos femininos ocorreram na população com 80 anos e mais de idade, sendo que os óbitos masculinos concentraram-se na população entre 60 e 79 anos (53\%). Importante diferença entre os sexos observa-se na faixa etária de 40 a 59 anos, onde ocorreram $64 \%$ mais óbitos masculinos (Tabela 2).

$\mathrm{Na}$ análise de correlação para o conjunto dos municípios, encontrou-se relação direta entre o CMM e as variáveis "Despesa em saúde por habitante/ano" "Percentual de internações por DAC". $\mathrm{Na}$ estratificação por população, considerando

Tabela 1. Maior e menor coeficiente médio de mortalidade e número de óbitos por doenças cardiovasculares em municípios selecionados, no período de 2003 a 2007 -16 Regional de Saúde/ RS.

\begin{tabular}{lcccc}
\hline No habitantes & Maior CMM & No de óbitos & Menor CMM & No de óbitos \\
\hline Até 5000 & 409,00 & 38 & 85,23 & 19 \\
5001 a 15.000 & 235,80 & 138 & 110,82 & 45 \\
Mais de 15.000 & 221,42 & 310 & 142,70 & 207 \\
\hline
\end{tabular}

Fonte: SIM/Datasus/MS e IBGE(2000). CMM: coeficiente médio de mortalidade por DCV.

Tabela 2. Número de óbitos e coeficiente de mortalidade por doenças cardiovasculares por faixa etária e sexo, no período de 2003 a 2007-16ª Regional de Saúde/RS.

\begin{tabular}{|c|c|c|c|c|c|c|c|c|c|c|}
\hline \multirow[b]{2}{*}{ Sexo } & \multicolumn{2}{|c|}{20 a 39 anos } & \multicolumn{2}{|c|}{40 a 59 anos } & \multicolumn{2}{|c|}{60 a 79 anos } & \multicolumn{2}{|c|}{80 anos e mais } & \multicolumn{2}{|c|}{ Total } \\
\hline & $\mathbf{n}$ & $\%$ & $\mathbf{n}$ & $\%$ & $\mathrm{n}$ & $\%$ & n & $\%$ & $\mathrm{n}$ & $\%$ \\
\hline Feminino & 16 & 1 & 151 & 10 & 628 & 41 & 727 & 48 & 1522 & 100 \\
\hline Masculino & 12 & 1 & 248 & 17 & 755 & 53 & 406 & 29 & 1421 & 100 \\
\hline Total & 28 & 1,81 & 399 & 25,85 & 1383 & 89,63 & 1133 & 73,42 & 2943 & 100 \\
\hline
\end{tabular}

Fonte: SIM/Datasus/MS e IBGE(2000). n: número de óbitos. 
municípios até 5000 habitantes, encontrou-se relação inversa entre o CMM e a "Distância em Km" entre o município e o serviço de referência em cardiologia e inversa, porém fraca $(\mathrm{p}<0,06)$ com a "Cobertura da ESF". Nos municípios entre 5000 e 15 mil habitantes encontrou-se relação direta entre o CMM e o IDESE e nos acima de 15 mil habitantes, observou-se relação inversa com a "Renda per capita" e relação direta com a "Distância em Km" e com o "Percentual de internações por DAC” (Tabela 3 ).

Todas as outras variáveis analisadas (Proporção de analfabetismo na população de 7 anos e mais, Proporção de população maior de 25 anos com mais de 12 anos de escolaridade, Proporção de população rural e Investimentos próprios em saúde) não apresentaram correlação com o CMM.

$\mathrm{Na}$ análise de regressão múltipla permaneceu significante apenas a relação com "Despesa em saúde por habitante/ano" e o coeficiente de determinação ( $\mathrm{R}^{2}$ ajustado) indicou que as variáveis estudadas explicam apenas $26 \%$ do desfecho, ou seja, do CMM.

\section{Discussão}

A utilização de dados secundários neste estudo é fator limitador dos resultados, considerando que as informações podem não ser totalmente fidedignas e que representam médias populacionais, já que se trata de um estudo ecológico.

Embora a distribuição do CM por sexo tenha se apresentado equiparada, talvez esta dis- tribuição fosse diferente se a avaliação tivesse sido feita por subgrupo (IAM e doenças cerebrovasculares). No entanto, a doença cardiovascular é a principal causa de morte para ambos os sexos, sendo que o número de mortes por estas doenças vem diminuindo nos homens e aumentando nas mulheres nas últimas décadas, principalmente a doença isquêmica do coração ${ }^{19}$. Estudo realizado em uma cidade de Portugal mostrou maior prevalência de fatores de risco para doenças cardiovasculares em homens do que em mulheres. Em relação a estas, a pesquisa revelou que em mulheres de classe socioeconômica mais baixa, há maior prevalência de fatores de risco ${ }^{20}$.

Em relação à distribuição por faixa etária, preocupa os óbitos de homens jovens (40 a 59 anos) e a concentração destes, abaixo dos 80 anos. Em regiões metropolitanas do Brasil, cerca de $50 \%$ dos óbitos masculinos por doença arterial coronariana (DAC) ocorrem na faixa etária abaixo de 65 anos, enquanto em outros países (Estados Unidos, Cuba e Inglaterra) essa proporção encontra-se em torno de $25 \%{ }^{21}$.

Os dados encontrados nesse estudo permitem fazer algumas considerações e levantar alguns questionamentos. Quando se exclui o efeito da diferença no tamanho da população, embora pequena, os resultados encontrados se modificam, trazendo a especificidade dos municípios muito pequenos, onde a cobertura da ESF pode representar um fator protetor melhor do que nos municípios maiores; e onde a renda per capita tem menor influência na mortalidade, pois se encontrou apenas nos municípios acima de 15 mil hab. relação inversa entre a renda per capita

Tabela 3. Correlação entre variáveis socioeconômicas, de cobertura e investimento com o coeficiente médio de mortalidade por doenças cardiovasculares - 16 ${ }^{\text {a }}$ Regional de Saúde/RS.

\begin{tabular}{lcccc}
\hline \multicolumn{1}{c}{ Variáveis } & CMM total & $\begin{array}{c}\text { CMM } \\
\text { Até } \mathbf{5 0 0 0} \text { hab }\end{array}$ & $\begin{array}{c}\text { CMM } \\
\mathbf{5 0 0 1} \mathbf{a} \mathbf{1 5 . 0 0 0} \text { hab }\end{array}$ & $\begin{array}{c}\text { CMM } \\
\text { mais de } \mathbf{1 5 . 0 0 0} \text { hab }\end{array}$ \\
\hline IDESE & $-0,09$ & $-0,00$ & $0,60^{*}$ & $-0,72$ \\
População Rural \% & 0,85 & $-0,13$ & $-0,29$ & 0,20 \\
Renda per Capita & $-0,17$ & $-0,23$ & 0,47 & $-0,94^{* *}$ \\
Despesa/habitante & $0,30^{*}$ & 0,08 & 0,42 & 0,51 \\
Invest. próprio em saúde & $-0,05$ & $-0,15$ & 0,03 & 0,52 \\
Distância em Km & $-0,30$ & $-0,62^{* *}$ & $-0,37$ & $0,88^{*}$ \\
Cobertura ESF & $-0,10$ & $-0,37$ & 0,24 & $-0,37$ \\
\% de analfabetismo & $-0,20$ & $-0,32$ & $-0,46$ & 0,66 \\
\% Internações DAC & $0,36^{*}$ & 0,34 & 0,20 & $0,83^{*}$ \\
\% mais de 12 anos escolaridade & $-0,23$ & $-0,01$ & 0,40 & -0.76 \\
\hline
\end{tabular}

Fonte: SIM/Datasus/MS, PNUD (2000), CNM (2000), FEE(2006), ABCR (2010). ${ }^{\star} \mathrm{p}<0,05 ;{ }^{* *} \mathrm{p}<0,01$. CMM: coeficiente médio de mortalidade por DCV. IDESE: Índice de desenvolvimento socioeconômico. DAC: doenças do aparelho circulatório 
e a mortalidade por DCV. Estes achados levam a hipótese de que nos municípios muito pequenos haja maior equanimidade na atenção à saúde.

Conforme Malta et al. ${ }^{22}$ a morte evitável pode derivar indicadores sensíveis à qualidade da atenção à saúde prestada pelo sistema de saúde, podendo levar a tomada de medidas de resultado ou de impacto desta atenção. Estes autores desenvolveram uma lista de causas de mortes evitáveis por intervenções do SUS, observando as tecnologias disponíveis neste sistema, incluindo nas causas de mortes evitáveis em maiores de 5 até 75 anos de idade, por ações adequadas de promoção à saúde, prevenção, controle e atenção às doenças não transmissíveis, doença hipertensiva, doença isquêmica do coração, insuficiência cardíaca e hemorragia intracerebral ou oclusão.

Outro achado foi a relação direta entre o percentual de internações por DAC e o CMM, principalmente nos municípios maiores. Esse dado, juntamente com o da regressão múltipla, que mostra a despesa em saúde por habitante/ano como o fator estatisticamente relacionado com o aumento do CMM, levanta a hipótese de que os recursos financeiros podem não estar sendo utilizados adequadamente, e estarem sendo direcionados predominantemente para internações, que são dispendiosas e nem sempre resolutivas.

As DCV estão incluídas na lista nacional de condições sensíveis à atenção primária, desenvolvida por Alfradique et al. ${ }^{23}$. Segundo estes autores, "altas taxas de internações por condições sensíveis à atenção primária em uma população, ou subgrupo(s) desta, podem indicar sérios problemas de acesso ao sistema de saúde ou de seu desempenho" 23 . No caso das doenças crônicas, estas internações podem indicar barreiras de acesso à atenção primária ou outros serviços de saúde, ou ainda manejo inadequado, não prevenindo a enfermidade e a hospitalização.

A Associação Nacional de Diretores das Doenças Crônicas (NACDD), nos Estados Unidos, desenvolveu um estudo sobre as competências necessárias aos profissionais de saúde pública para o controle e a prevenção das doenças crônicas. Destacaram-se as de comunicação e as habilidades culturais, assim como gerenciais, que incluem: conduzir estratégias, gerenciar pessoas, programas e recursos, projetar e avaliar programas, usar a ciência da saúde pública, influenciar políticas e alteração de sistemas, e construir suporte ${ }^{24}$.

Estudo realizado por Ishitani et al. ${ }^{14}$ em 98 municípios com mais de 100 mil habitantes revelou associação inversa entre condições socioeconômicas (renda, escolaridade e moradia) e mor- talidade por DCV, destacando-se a escolaridade, e relação direta com taxa de pobreza. No entanto, o valor baixo do coeficiente de determinação ( $\mathrm{R}^{2}$ ajustado) encontrado no presente estudo, sugere que outras variáveis, além das pesquisadas, podem estar associadas à mortalidade por DCV. Meta-análise realizada para verificar a relação entre condições socioeconômicas e Infarto Agudo do Miocárdio (IAM), observou risco aumentado para IAM em grupos de menor condição socioeconômica em relação à renda, ocupação e educação. No entanto, este risco é claro em países ricos, como Estados Unidos, Canadá e Europa, mas o resultado é inconsistente em regiões de média e baixa renda ${ }^{25}$. Este resultado coincide com o encontrado no nosso estudo, realizado em uma região de renda média.

A distância entre o município e o serviço de referência regional só mostrou-se relevante nos municípios com mais de 15 mil habitantes, sendo que nos menores até aparece com relação inversa, não ficando clara sua influência na mortalidade por DCV, possivelmente devido ao pequeno tamanho da amostra.

Os resultados do presente estudo mostraram a necessidade de aprofundamento da questão, tendo em vista que embora as condições sejam muito semelhantes entre os municípios, as diferenças nos indicadores são expressivas. O estudo revelou que as variáveis socioeconômicas, de investimento, de cobertura e de acesso selecionadas não conseguem explicar porque municípios com condições muito semelhantes apresentam diferenças nos resultados de mortalidade por DCV, que chegaram a ser até de 4,8 vezes, entre o maior e o menor CMM.

Os Anais da Conferência sobre Status Socioeconômico e Saúde, da Academia de Ciências de Nova Iorque, referem que não há consenso entre a melhor forma de medir os diversos componentes deste status (educação, renda, profissão, entre outros). Seus estudos têm concluído que a ligação entre status socioeconômico e morbidade e mortalidade é complexa. Outra dificuldade além da aferição dos indicadores são as diferenças entre grupos sociodemográficos ${ }^{26}$. Pesquisa realizada com dados da coorte Gazel, na França, buscou conhecer em que medida os fatores de risco mediavam as diferenças verificadas na incidência de doença coronariana entre grupos socioeconômicos diferentes, sendo maior nos grupos de menor condição socioeconômica. Verificou-se que fatores tradicionais de risco de DAC, embora fortemente associados à doença coronariana, explicavam apenas uma pequena parte 
da associação entre posição socioeconômica e incidência de doença coronariana. Tabagismo e colesterol apresentaram papel um pouco maior de mediadores do que outros fatores de risco ${ }^{27}$.

Carvalho e Cunha ${ }^{28}$ referem que diferentes serviços de saúde ou municípios, com a mesma quantidade de recursos, podem produzir resultados absolutamente diversos, dependendo como articulam estes recursos. Afirmam que a compreensão que se tem sobre saúde e doença condiciona o olhar sobre a realidade definindo o que é problema de saúde e assumindo papel determinante sobre as características organizativas do setor da saúde.

Estudo de Arretche e Marques ${ }^{29}$ concluiu que o sistema público de saúde permitiu a generalização da transferência das funções de oferta de serviços básicos de saúde para os municípios, mas não produziu equalizações nos padrões de oferta destes serviços. As regras que orientaram as transferências federais estiveram mais orientadas para a efetivação da descentralização do que para a promoção da redução das desigualdades entre os municípios.

Considera-se que outros olhares sobre este fenômeno sejam necessários, pois a maioria dos pequenos municípios encontra-se em situação de grande dependência externa em relação a referências de média e alta complexidade, com algumas dificuldades de acesso a estes serviços e diferentes formas de organização da atenção básica e na estrutura dos serviços de saúde. Conforme a $\mathrm{OPAS}^{30}$ a municipalização tem avançado, mas subsistem alguns problemas derivados do pequeno porte da maioria dos municípios brasileiros, o que afeta a racionalidade de escala e compromete a eficiência do sistema de saúde, em termos de resolutividade e qualidade.

O grande desafio está em garantir a equidade e a integralidade do acesso e da atenção à saúde da população de municípios com pequena infraestrutura de serviços, principalmente naqueles com poucos habitantes.

\section{Colaboradores}

CRG Medeiros trabalhou na concepção, delineamento, coleta, análise, interpretação de dados e redação final do artigo. SN Meneghel e TE Gerhardt participaram da concepção, delineamento e interpretação dos dados. 


\section{Referências}

1. Organización Panamericana de la Salud (OPAS). Serie La Renovación de la Atención Primaria de Salud en las Américas. Redes Integradas de Servicios de Salud. Conceptos, Opciones de Política e Hoja de Ruta para su Implementación en las Américas. 2008 [site na Internet]. [acessado 2011 jan 13]. Disponível em: http://portal.saude.gov.br/portal/arquivos/pdf/doc_opas_espanhol.pdf.

2. Mendes EV. As redes de atenção à saúde. Rev Med Minas Gerais 2008; 18(Supl 4):S3-S11.

3. Organización Panamericana de la Salud (OPAS). Rede Interagencial de Informação para a Saúde (Ripsa). Indicadores básicos para a saúde no Brasil. 2a Edição. Brasília: OPAS; 2008.

4. Instituto Brasileiro de Geografia e Estatística. [acessado 2011 jul 30]. Disponível em: http://www. ibge.gov.br/home/estatistica/populacao/censo2010/ tabelas_pdf/Rio_grande_do_sul.pdf

5. Brasil. Ministério da Saúde (MS). Brasília: Informações em Saúde. [internet]. [acessado $2010 \mathrm{abr}$ 25]. Disponível em: http://www.datasus.gov.br/datasus/datasus.php

6. Eyken EBBDV, Moraes CL. Prevalência de fatores de risco para doenças cardiovasculares entre homens de uma população urbana do Sudeste do Brasil. Cad Saude Publica 2009; 25(1):111-123.

7. Capilheira MF, Santos IS, Azevedo Júnior MR, Reichert FF. Risk factors for chronic non-communicable diseases and the CARMEN Initiative: a population-based study in the South of Brazil. Cad Saude Publica 2008; 24(12):2767-2774.

8. Rezende EM, Sampaio IBM, Ishitani LH. Causas múltiplas de morte por doenças crônico-degenerativas. Cad Saude Publica 2004; 20(5):1223-1231.

9. Vieira da Silva LMV. Avaliação do processo de descentralização das ações de saúde. Cien Saude Colet 1999; 4(2):331-339.

10. Buss PM, Pellegrini Filho A. A saúde e seus determinantes sociais. Physis 2007; 17(1):77-93.

11. Comissão Nacional sobre Determinantes Sociais da Saúde. As causas sociais das iniqüidades de saúde no Brasil. Relatório final. Rio de Janeiro: Fiocruz; 2008.

12. Barros MBA, César CLG, Carandina L, Torre GD. Desigualdades sociais na prevalência de doenças crônicas no Brasil, PNAD 2003. Cien Saude Colet 2006; 11(4):911-926.

13. Da Costa JS, Barcellos FC, Sclowitz ML, Sclowitz IK, Castanheira M, Olinto MT, Menezes AM, Gigante DP, Macedo S, Fuchs SC. Prevalência de Hipertensão Arterial em Adultos e Fatores Associados: um Estudo de Base Populacional Urbana em Pelotas, Rio Grande do Sul, Brasil. Arq Bras Cardiol. 2007; 88(1):59-65.

14. Dachs JNW. Determinantes das desigualdades na auto-avaliação do estado de saúde no Brasil: análise dos dados da PNAD/1998. Cien Saude Colet 2002; 7(4):641-657.
15. Ishitani LH, Franco GC, Perpétuo IHO, França E. Desigualdade social e mortalidade precoce por doenças cardiovasculares no Brasil. Rev Saude Publica 2006; 40(4):684-691.

16. Fundação de Economia e Estatística (FEE). Rio Grande do Sul. Índice de Desenvolvimento Socioeconômico (IDESE). [internet]. [acessado 2010 abr 25]. Disponível em: http://www.fee.tche.br/sitefee/pt/ content/estatisticas/pg_idese.php

17. Associação Brasileira de Concessionárias de Rodovias [internet]. São Paulo: Distância entre cidades [acessado 2010 Abr 25]. Disponível em: http://www. abcr.org.br/geode/index.php

18. Confederação Nacional dos Municípios. [acessado 2010 Dez 10]. Disponível em: http://www.cnm. org.br/index.php?option $=$ com_wrapper\&view $=$ wrapper\&Itemid $=94$

19. Programa das Nações Unidas para o Desenvolvimento. [acesso 2010 Dez 15]. Disponível em: http:/ /www.pnud.org.br/IDH/Atlas2003.aspx?indice Accordion $=1 \&$ li=li_Atlas2003

20. Finato VML, Nakazone S, França, HH, Aspectos típicos da cardiopatia isquêmica no sexo feminino, Revista Fac. Ciênc. Méd 2005; 7(4):6-9.

21. Alves L, Azevedo A, Silva S, Barros H. Socioeconomic Inequalities in the Prevalence of Nine Established Cardiovascular Risk Factors in a Southern European Population. PLoS ONE 2012; 7(5):e37158. Published online 2012 May 29. [acessado 2012 ago 3]. Disponível em: http://www.plosone.org/article/ info\%3Adoi\%2F10.1371\%2Fjournal.pone.0037158

22. Avezum A, Piegas LS, Pereira JCR. Fatores de risco associados com Infarto Agudo do Miocárdio na região metropolitana de São Paulo: Uma região desenvolvida em um país em desenvolvimento. Arquivos Brasileiros de Cardiologia 2005; 84(3):206-213.

23. Malta, DC, Duarte EC, Almeida MF, Dias MAS, Morais Neto, OL, Moura L, Ferraz, W, Souza, MFM. Lista de causas de mortes evitáveis por intervenções do Sistema Único de Saúde do Brasil. Epidemiol. Serv. Saúde 2007; 16(4):233-244.

24. Alfradique MA, Bonolo PF, Dourado I, Lima-Costa MF, Macinko J, Mendonça CS, Oliveira VB, Sampaio LFR, De Simoni C, Turci MA. Internações por condições sensíveis à atenção primária: a construção da lista brasileira como ferramenta para medir o desempenho do sistema de saúde (Projeto ICSAP - Brasil). Cad Saude Publica 2009; 25(6):1337-1349.

25. Slonim A, Wheeler FC, Quinlan KM, Smith SM. Designing competencies for chronic disease practice. Prev Chronic Dis 2010; 7(2):A44. [acessado 2012 ago 02]. Disponível em: http://www.cdc.gov/pcd/ issues $/ 2010 / \mathrm{mar} / 08$ 0114.htm

26. Manrique-Garcia E. Socioeconomic position and incidence of acute myocardial infarction: a metaanalysis. J Epidemiol Community Health 2011; 65(4): 301-309. 
27. Adler NE, Stewart J. Preface to the biology of disadvantage: socioeconomic status and health. Ann NY Acad Sci 2010; 1186:1-4.

28. Silhol R, Zins M, Chauvin P, Chaix B. Investigating the spatial variability in incidence of coronary heart disease in the Gazel cohort: the impact of area socioeconomic position and mediating role of risk factors. J Epidemiol Community Health 2011; 65(2): 137-143.

29. Carvalho SR, Cunha GT. A gestão da atenção na saúde: elementos para se pensar a mudança da organização na saúde. In: Campos GWS, organizador. Tratado de Saúde Coletiva. São Paulo: Hucitec; 2006. p. 837-868.

30. Arretche M, Marques E. Condicionantes locais da descentralização das políticas de saúde. In: Hochman G, Arretche M, Marques E, organizadores. Políticas públicas no Brasil. Rio de Janeiro: Editora Fiocruz; 2007. p. 173-204.

31. Organização Pan-Americana da Saúde. A atenção à saúde coordenada pela APS: construindo as redes de atenção no SUS: contribuições para o debate. Brasília: OPAS; 2011.

Artigo apresentado em 30/04/2012

Aprovado em 17/07/1012

Versão final apresentada em 30/08/2012 